\title{
Mixed type endometrial cancer, a series of six cases.
}

Amira Badawy, MD,

Ass. Prof. of obstetrics \& gynaecology, Alexandria Gyne-Oncology Group (AGOG),

Gyne-oncology unit, department of obstetrics \& gynaecology, Faculty of Medicine,

Alexandria University, Egypt.

\section{INTRODUCTION:}

Endometrial cancer is the most common malignant tumour of the female genital tract, Type-1 (endometrioid) endometrial adenocarcinoma is the pathological variant detected in the vast majority of cases, while type-2 (nonendometrioid) endometrial adenocarcinoma is less common but usually more aggressive and of high grade. Type-2 endometrial adenocarcinoma includes papillary serous and clear cell carcinoma, in addition to carcino-sarcoma (malignant mixed Mullerian tumours) varieties. Endometrial stromal sarcoma is a very rare type of endometrial cancer, representing only $0.2 \%$ of all uterine malignancies.

The presence of mixed pathologic patterns of gynaecologic cancers is not a common finding. In endometrial cancer, mixed epithelial carcinoma is defined as a tumour composed of two or more different histological types of endometrial carcinoma, at least one of which is of the type II category, with at least $10 \%$ of each component. It is also called mixed cell adenocarcinoma.

Coexistence of endometrial stromal sarcoma and endometrial epithelial carcinoma is an extremely rare finding.

\section{PATIENTS \& PROCEDURES:}

In the current study, cases of endometrial cancer were revised during a 24 months period of time (2016 \& 2017). Six cases of mixed endometrial cancers were found, five of them had mixed adenocarcinoma (i.e. mixed epithelial carcinoma, Typ-1 and Type-2), while the sixth case had mixed type-1 adenocarcinoma and endometrial stromal sarcoma.

All cases were subjected to full surgical staging, including total hysterectomy with bilateral salpingo-oophorectomy, ascitec (or peritneal wash) sampling, total omentectomy, peritoneal biopsies and lymph-adenectomy.

\section{DISCUSSION:}

The details of cases are presented in the table below. It was noted that non-endometrioid element was predominant in most of mixed epithelial carcinoma cases (4 out of 5).

The sixth case which was a mixed type-1 endometrioid adenocarcinoma and endometrial stromal sarcoma is one of the rarest pathologic combinations reported world wide, and this one is considered the $6^{\text {th }}$ published case up till now.

Case number (5) had a very deceiving presentation compared to the aggressive nature of the tumour. The patient (65 years old) was presented with an acute attack of lower abdominal pain only. Pelvic ultrasound and MRI showed only endometrial polyp with minimal free fluid within the cavity, thin endometrium, no enlarged lymph nodes or myometrial invasion. Malignancy was confirmed after endometrial sampling then staging laparotomy was performed, where omentum showed localized areas of thickening with fine nodularity affecting the under surface of the right diaphragmatic copula. Pathologically the endometrial polyp was harbouring a high grade endometrioid carcinoma, while the serous element was arising from the remaining atrophic endometrium with very extensive affection of the lymphovascular spaces and metastasis to the right adnexa, omentum and peritoneum (see figure below).

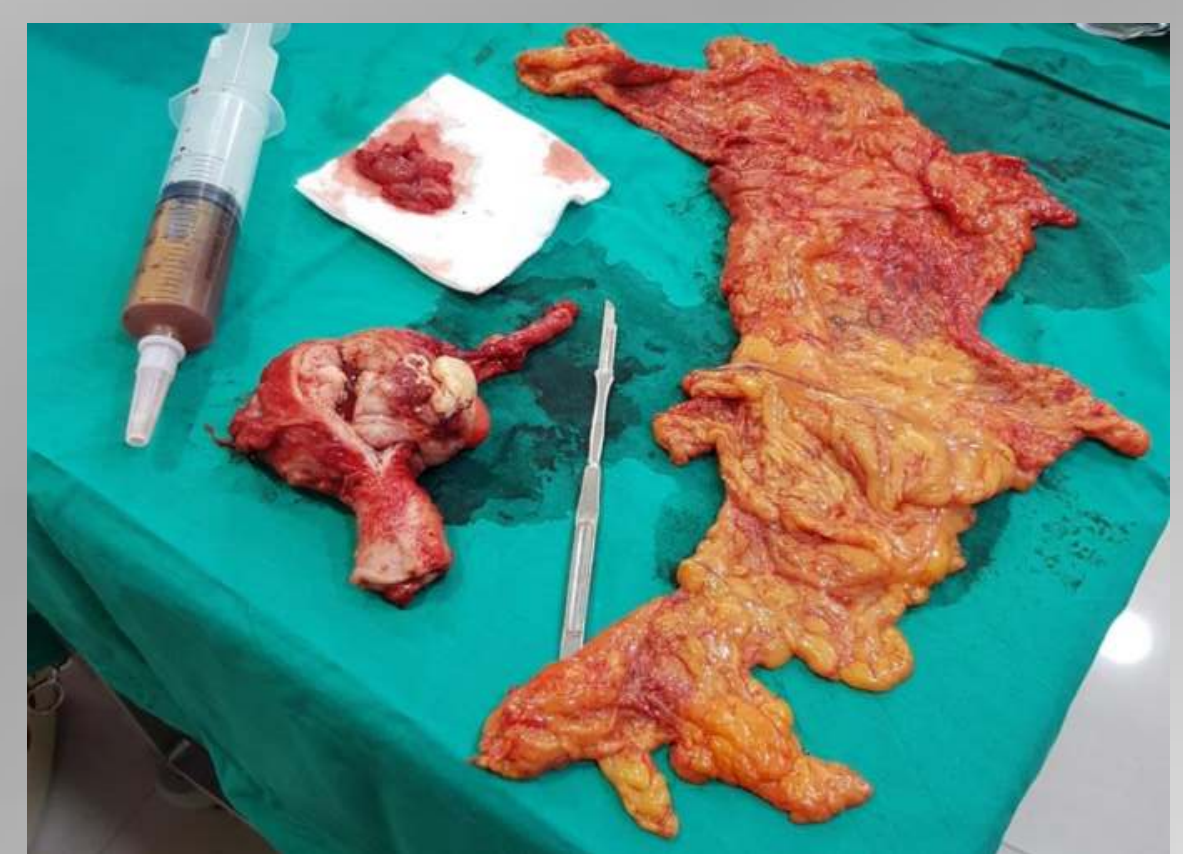

Case number (5): A Figure showing, surgical staging of a case of advanced (stage IVb) mixed endometrioid adenocarcinoma and serous adenocarcinoma.

\begin{tabular}{|c|c|c|c|c|c|c|}
\hline \multirow{2}{*}{$\begin{array}{l}\text { Case } \\
\text { № }\end{array}$} & \multirow{2}{*}{$\begin{array}{l}\text { Age in } \\
\text { years }\end{array}$} & \multirow{2}{*}{$\begin{array}{l}\text { Presenting symptom } \\
\text { (and duration) }\end{array}$} & \multicolumn{2}{|c|}{ Pathologic variants (and average $\%$ of affection) } & \multirow{2}{*}{ Gr } & \multirow{2}{*}{ St } \\
\hline & & & $1^{\text {st }}$ variant & $2^{\text {nd }}$ variant & & \\
\hline 1 & 60 & PMUB (6 months). & - EEA $40 \%$. & - Carcino-sarcoma $60 \%$. & 3 & $\mathrm{lb}$ \\
\hline 2 & 60 & PMUB + lower abdominal pain (6 months). & - EEA $40 \%$. & - Serous adenocarcinoma $60 \%$. & 2 & III \\
\hline 3 & 62 & PMUB (3 months). & - EEA $20 \%$ & - Clear cell adenocarcinoma $80 \%$. & 2 & la \\
\hline 4 & 70 & PMUB (10 months). & - EEA $70 \%$ & - Serous adenocarcinoma $30 \%$. & 2 & II \\
\hline 5 & 65 & Acute attack of pelvic pain (5 days). & - EEA $30 \%$ & - Serous adenocarcinoma $70 \%$. & 3 & $\mathrm{IVb}$ \\
\hline & 67 & PMUB + abdominal pain (4 months). & - EEA $60 \%$. & - Endometrial stromal sarcoma $40 \%$. & 2 & III \\
\hline
\end{tabular}

Table showing the details of cases of mixed endometrial cancer reported by the current study
PMUB : post-menopausal uterine bleeding. EEA : endometrioid endometrial adenocarcinoma. Gr : Grade.

St : Stage.
ESGO state of art conference 2018 Author: Amira Badawy.

Email: dr.amirabadawy@gmail.com Tel: 002-01148087666 